\title{
Epidemiology of gliomas in women diagnosed with breast cancer supports the protective role of estrogenic exposure
}

\author{
Mezencev R \\ Georgia Institute of Technology, School of Biological Sciences, Atlanta, GA, USA. \\ roman.mezencev@biosci.gatech.edu
}

\begin{abstract}
Gliomas represent the most common type of brain cancers, but their etiology is still poorly understood and understanding of their risk factors is limited. The role of sex hormones in the development of brain cancers has been suggested by epidemiological and experimental studies and clinical observations, which highlighted the need for more evidence before hormone-modulating interventions are developed for prevention or treatment of gliomas. The results of this retrospective cohort study using data from SEER 9 cancer registries demonstrate a decreased risk of brain cancers in women diagnosed with breast cancers at 40+ years, which is consistent with hypothesis that estrogenic exposure reduces the risk of development of gliomas. In contrast, women diagnosed with breast cancer at $<40$ years display an increased risk of subsequent brain cancers than women in general population, which likely reflects the role of shared genetic risk factors. These findings support the protective role of estrogens against development of gliomas and suggest the importance of early diagnosis and development of preventive approaches for women diagnosed at younger age (Tab. 2, Fig. 1, Ref. 37). Text in PDF www.elis.sk. KEY WORDS: gliomas in women, breast cancer, estrogenic exposure.
\end{abstract}

\section{Introduction}

Second primary tumors are serious events experienced by cancer survivors and they represent the leading cause of death in some populations of long-term cancer survivors (1). For this reason, analysis of the risk of developing second primary cancers can improve risk-informed health care of cancer survivors. In addition, multiple primary cancers can be related through shared genetic susceptibility, environmental, lifestyle or iatrogenic exposures (2), and so the insight from epidemiology of second primary cancers can contribute to better understanding of the risk factors associated with specific cancers (2).

Primary malignant brain tumors are a heterogeneous group of diseases diagnosed annually in more than 250000 people worldwide. About $77 \%$ of them represent gliomas, which include astrocytic, oligodendroglial, and ependymal tumors and mixed gliomas. The most commonly diagnosed glioma in children and young adults is pilocytic astrocytoma, which accounts for $37 \%$ of gliomas diagnosed under the age of 20 years. In contrast, the most common glioma in adults is glioblastoma that represents 67 $\%$ of the adult glioma cases (3). Glioblastoma can be diagnosed at any age, and prognosis for patients diagnosed with glioblastoma is generally poor. Depending on the age at diagnosis, 2-year relative

Georgia Institute of Technology, School of Biological Sciences, 310 Ferst Dr., Atlanta, GA, USA.

Address for correspondence: R. Mezencev, PhD, Georgia Institute of Technology, School of Biological Sciences, 310 Ferst DR, Atlanta, GA 30329, USA survival of glioblastoma patients ranges from $3.1 \%$ ( $>75$ years) to $32.6 \%$ (<20 years) (3). Prevention of glioblastoma, and brain cancers in general, would benefit from identification of modifiable risk factors. However, this effort has not been successful, except for identification of high-doses of ionizing radiation as the only established modifiable risk factor for several types of brain cancers (4). This is caused, at least in part, by considerable heterogeneity of brain cancers, diagnostic challenges, as well as methodological limitations of epidemiological studies.

Sex hormones have been hypothesized to play a role in development of gliomas and meningiomas. Results of several experimental and epidemiological studies, as well as clinical observations, suggested that estrogens and progestins support the development and progression of meningiomas and impair prognosis of meningioma patients. In contrast, results of some in vitro, animal and epidemiological studies implied a protective role of estrogens against gliomas (reviewed in (5)).

Possible role of the exogenous or endogenous exposure to estrogens in meningioma was further supported by epidemiological studies of multiple primary cancers. This is specifically relevant to multiple primaries that included breast cancers, which represent a malignancy with a clearly established role of estrogens. Intriguingly, women with breast cancers were shown to display a higher incidence of meningioma, and similarly, women diagnosed with meningioma demonstrated a higher risk of development of breast cancer. This bi-directional positive association between breast cancers and meningioma implies with a higher confidence that these two malignancies are associated through a shared risk factor, such as an exposure to estrogens. Nevertheless, this association could 
also be explained alternatively, by shared risk factor of genetic nature, such as: polymorphism of the BRIP1 gene, which was previously associated with both meningiomas (6) and breast cancers (7).

In contrast to meningioma, the association between breast cancer and gliomas have been studied or reported only in limited context, and available reports suggested, contrary to the expectations, that women diagnosed with breast cancer displayed an increased risk of development of gliomas or, more specifically, glioblastoma (8). In this report, the association between breast cancers and brain cancers represented by gliomas (and mostly by glioblastoma) was evaluated by a retrospective cohort study, using the data reported by high-quality population-based SEER Cancer Registries. The results of this study demonstrated that breast cancer diagnosed in younger but not in older women increased the risk of subsequent development of brain cancers. In older women, diagnosis of breast cancer is associated with a decreased risk of brain cancers, and likewise, women with history of glioblastoma are less likely to develop breast cancers than women in general population, which supports the hypothesis that exposure to estrogen is a negative risk factor for gliomas.

\section{Materials and methods}

Database: this analysis included cases reported to nine population-based cancer registries (Incidence - SEER 9 Registries Research Data) between January 1973 and December 2014 (release date: April 2017). The SEER 9 Registries cover approximately 9.4 $\%$ of the U.S. population (http://seer.cancer.gov/registries/data. html) and the database released on April 2017 contains records on 5037307 tumors (http://seer.cancer.gov/data/seerstat/nov2016/). Population-based cancer registries are an appropriate resource for evaluation of the risk of subsequent primary cancers because of large number of recorded survivors, long follow-up times and standardized registry methods (9).

The analysis of the risk of brain cancers after diagnosis of breast cancer was restricted to women diagnosed with invasive duct carcinoma of no special type (NST), ICD-O-3: 8500/3, which accounts for $\sim 80 \%$ of all invasive breast cancers (10). Since breast cancer risk factors, tumor biology and prognosis are different between young women below 40 years and older women (11), the risk of second primary brain cancers following primary breast cancer was determined separately for women aged 0-39 and 40+ years. Additionally, the risk of second primary brain cancers was evaluated separately for women, who did not receive radiotherapy and those, who received radiotherapy for breast cancer (beam radiation, radioactive implants or combination modalities that included beam radiation with implants or isotopes).

Women diagnosed with infiltrating duct carcinoma of the breast as their first tumor were included into the index cohort and followed for the occurrence of subsequent primary brain cancers. The statistical methodology used for the determination of the risk of subsequent primary cancers was previously described (12). Briefly, the person-years at risk (PYR) for developing brain cancers were accumulated from 2 months after diagnosis of breast cancer to (i) date of death, (ii) date of last known vital status, or (iii) end of the study, whichever occurred first. The risk was determined as a standardized incidence ratio (SIR), which was calculated as $\mathrm{O} / \mathrm{E}$, where $\mathrm{O}$ is the number of observed and $\mathrm{E}$ is the number of expected primary brain cancer cases in the same cohort based on age-, race- and 5-year-period specific incidences in general population. SIR values were determined for follow-up periods (latencies) 0-59, 60-119 and 120+ months and for all intervals combined after diagnosis of breast cancers, and the $95 \%$ confidence intervals for SIR (CI95) were calculated using exact method (13). SIR values are reported as statistically significant if their CI95 intervals do not include 1.0 (the probability that the observed number of cases was significantly different than the expected due to chance alone was less than 0.05).

SIR and CI95 values were calculated using the NCI SEER*Stat software version 8.3.5 (seer.cancer.gov/seerstat) using the following settings: Rates: (Rate Name: SEER 9 1973-2014 (Nov 2016 sub), Race (WU/B/O)); Parameters: Latency Exclusion Period: 2 months; Events: Analysis Type: Single Outcome Analysis; Events: Brain; Exit Point: Exit at Events for Display. Statistic: Confidence Intervals: Exact Method. Multiple Primary Selection was set to "First Primary in Database" for analysis of the risk of breast cancers following glioblastoma, and "First Primary Only (Sequence Number 0 or 1)" for analysis of the risk of brain cancers following breast cancer.

Evaluation of the risk between two cancers following each other in both directions can help to interpret the association between two cancers, because significantly increased (or decreased) risk between two malignancies that are bi-directional in nature are suggestive of shared etiologic (or protective) factors (14). For this reason, the risk of primary breast cancer following glioblastoma was determined using the same statistical methodology and database as above.

\section{Results}

Women diagnosed with infiltrating duct carcinoma of the breast as their first tumor were identified $(\mathrm{N}=389415)$ and followed for $0-41.8$ years (median $=7.8$ years) for the occurrence of subsequent primary brain cancers. The total follow-up time was 3901837.49 person-years (the cohort characteristics are presented in Table 1). During the follow-up, 376 women developed brain cancers at median time of 80 months after the diagnosis of breast cancer (range 2-461 months). For these 376 patients, median age at diagnosis was 60 years for breast cancer (range: $21-88$ yrs) and 69 years for brain cancer (range: 24-96 yrs). The most prevalent histological type of brain cancer reported in this group was glioblastoma diagnosed in 250 women (66.5 \%; binomial CI95: 61.5-71.2\%).

The risk of developing subsequent brain cancer was significantly increased for women diagnosed with breast cancer before the age of 40 years, when all latency times were considered together $(\mathrm{SIR}=1.64 ;$ CI95 $=1.08-2.39 ; 27$ cases $)$. The risk was also increased for other latency intervals under consideration, but it did not reach statistical significance (Fig. 1A). In contrast, older women, who were diagnosed with breast cancer at the age of $\geq 40$ years, displayed a significantly decreased risk of subsequent brain 
Tab. 1. Characteristics of breast cancer cohort studied for subsequent occurrence of primary brain cancers ${ }^{\ddagger}$.

\begin{tabular}{lcc}
\hline & $\begin{array}{c}\text { Breast cancer cohort } \\
(\mathrm{n}=389415)\end{array}$ & $\begin{array}{c}\text { Subset that developed } \\
\text { brain cancers } \\
(\mathrm{n}=376)\end{array}$ \\
\hline Age at BC diagnosis & & \\
Median (range) & 60 yrs (15-107) & 60 yrs (21-88) \\
0-39 years & 27437 & 27 \\
40+ years & 361978 & 349 \\
\hline Grade of BC & & \\
Grade I & 48659 & 38 \\
Grade II & 116835 & 101 \\
Grade III & 112817 & 102 \\
Grade IV & 6115 & 9 \\
NA & 104989 & 5 \\
\hline Stage of BC & & 263 \\
Localized & 234029 & 104 \\
Regional & 130989 & 4 \\
Distant & 18513 & 126 \\
Unknown & 5884 & \\
\hline ₹C: breast cancer. Grade: Grade I (well differentiated), Grade II (moderately dif- \\
ferentiated), Grade III (poorly differentiated), Grade IV (undifferentiated; anaplas- \\
tic), NA: not determined, not stated or not applicable. For more information, see \\
https://seer.cancer.gov/data-software/documentation/seerstat/nov2016/TextData. \\
FileDescription.pdf\#pa Stage: SEER Historic Stage A: in situ, localized, regional, \\
distant, \& unknown. For more information, see http://seer.cancer.gov/seerstat/vari- \\
ables/seer/lrd-stage
\end{tabular}

Tab. 2. Characteristics of glioblastoma cohort studied for subsequent occurrence of primary breast cancers ${ }^{\mathrm{\#}}$.

\begin{tabular}{lcc}
\hline & $\begin{array}{c}\text { Glioblastoma } \\
\text { cohort } \\
(\mathrm{n}=9405)\end{array}$ & $\begin{array}{c}\text { Subset that developed } \\
\text { breast cancers } \\
(\mathrm{n}=12)\end{array}$ \\
\hline $\begin{array}{c}\text { Age at GB diagnosis } \\
\text { Median (range) }\end{array}$ & 63 yrs $(0-100)$ & 63 yrs $(43-90)$ \\
\hline Primary site of GB & & \\
C71.0 Cerebrum & 368 & 0 \\
C71.1 Frontal lobe & 2428 & 4 \\
C71.2 Temporal lobe & 2019 & 3 \\
C71.3 Parietal lobe & 1674 & 1 \\
C71.4 Occipital lobe & 357 & 1 \\
C71.5 Ventricle, NOS & 44 & 0 \\
C71.6 Cerebellum & 61 & 0 \\
C71.7 Brain stem & 83 & 0 \\
C71.8 Overlapping lesion & 1627 & 0 \\
C71.9 Brain, NOS & 713 & 3 \\
C72.0 Spinal cord & 26 & 0 \\
Other & 5 & 0 \\
\hline
\end{tabular}

${ }^{¥}$ GB: glioblastoma. Primary site: the site in which the primary tumor originated. For more information see For more information, see https://seer.cancer.gov/data-software/ documentation/seerstat/nov2016/TextData.FileDescription.pdf\#pa

cancers for all latencies combined $(\mathrm{SIR}=0.77$; CI95 $=0.69-0.86$; 349 cases) and for all specific latency intervals, except for period 2-11 months since the diagnosis of breast cancer (Fig. $1 \mathrm{~B}$ ).

To account for the possible effect of radiation therapy, which was administered to some breast cancer patients, the risk of subsequent brain cancers was determined separately for breast cancer patients that were known to have received radiotherapy $(\mathrm{n}=$ 169677) or no radiotherapy $(\mathrm{n}=210156)$. The results suggested that radiation therapy did not change the pattern of decreased risk of brain cancers in women diagnosed with breast cancer at $40+$ years. SIR values for all latency intervals combined were found
0.85 (CI95: 0.72-1.00; 149 cases) for women, who received radiotherapy and 0.72 (CI95: 0.62-0.83; 193 cases) for women not treated with radiotherapy (both statistically significant). Women diagnosed with breast cancer under the age of 40 displayed an increased risk of brain cancers regardless of radiotherapy status, but the risk was not statistically significant.
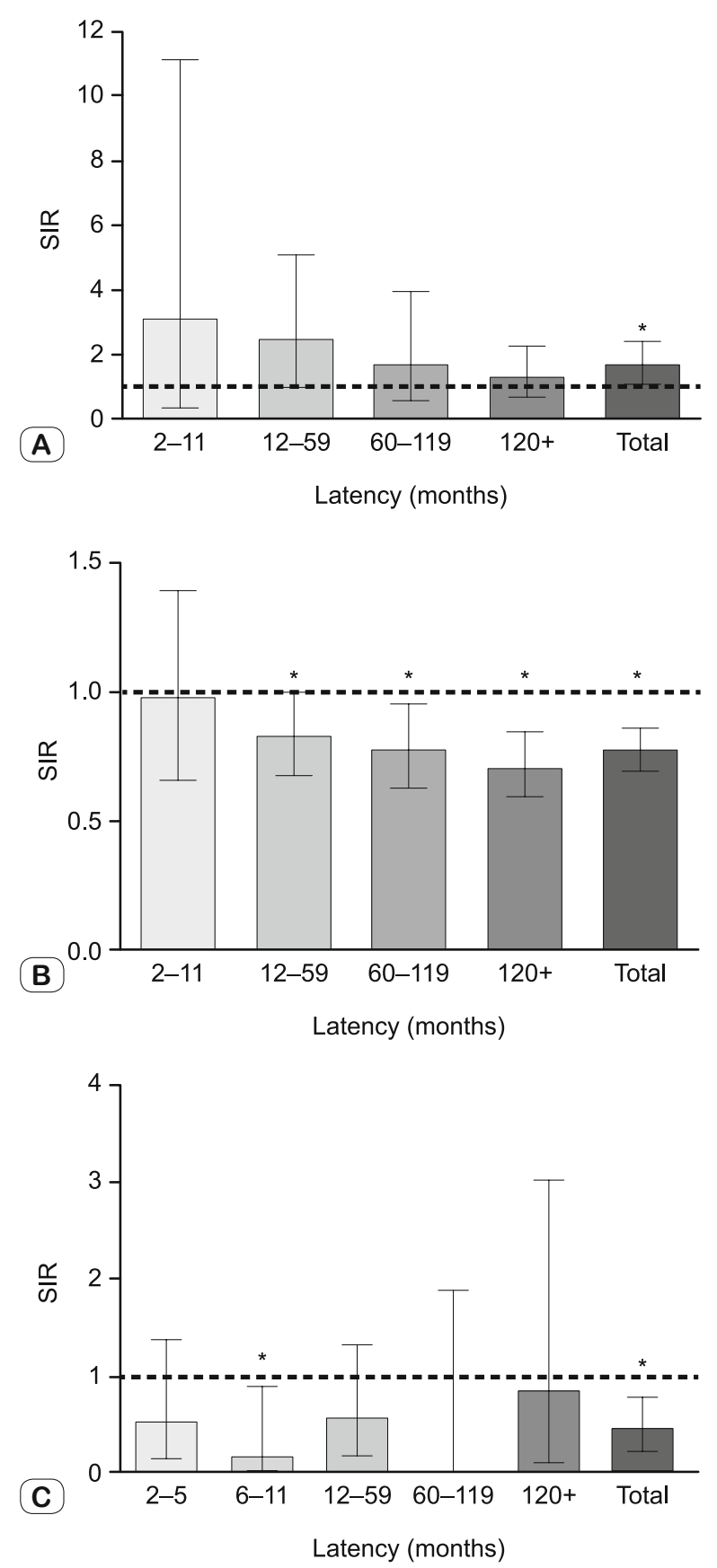

Fig. 1. Risk of developing: (A) brain cancers following breast cancers diagnosed in women aged < 40 years; (B) brain cancers following breast cancers diagnosed in women aged $40+$ years; $(C)$ breast cancers following glioblastoma diagnosis. SIR: Standardized Incidence Rate. Horizontal line represents risk equal to standard population (SIR = 1). Error bars: c/95. 
463-468

To examine whether the observed inverse association between the risk of breast cancers and brain cancers is bi-directional, this analysis was expanded to determinate the risk of developing subsequent primary breast cancers in women diagnosed with brain cancers as their first malignancies. This analysis was limited to women diagnosed with glioblastoma, because it is the most prevalent brain cancer in adults. The cohort included 9146 cases reported to the SEER 9 registry during time period 1973-2014, who were followed for $0-40.8$ years (median $=0.5$ year/person). Total accumulated follow-up time was 10420.96 person-years (the cohort characteristics are presented in Table 2). Of these women, 12 developed subsequent invasive breast cancer at median time 28 months since diagnosis (range: 2-223 months). Median age at their breast cancer diagnosis was 66 years (range 48-90) and histological types included (i) infiltrating duct carcinoma ( 9 cases), (ii) lobular carcinoma (1 case), (iii) infiltrating duct and lobular carcinoma ( 1 case), and in one case histopathological information was missing. Stage at breast cancer diagnosis was recorded as localized (10 cases), regional ( 1 case) and unknown ( 1 case). Calculated SIR values demonstrated that women previously diagnosed with glioblastoma had significantly lower risk of subsequent development of breast cancer during 6-11 months after breast cancer diagnosis ( $\mathrm{SIR}=0.16$; CI95: $0-0.88 ; 1$ case) and overall (SIR $=0.45 ;$ CI95 $=0.23-0.78 ; 12$ cases $)$. The risk of breast cancer in glioblastoma survivors was lower than in the general population for other latency intervals as well, but the risk reduction did not reach statistical significance (Fig. 1C).

\section{Discussion}

Etiology of gliomas is poorly understood. The most firmly established risk factors are advanced age, male sex, Caucasian race, as well as some genetic syndromes (e.g. Li-Fraumeni syndrome, neurofibromatosis type I and II, Turcot's syndromes, melanomaastrocytoma syndrome, BRCA syndrome) (15), and ionizing radiation (16). In addition, other environmental exposures and health or genetic factors have been reported as possibly associated with the risk of gliomas, including diet, beauty products, cell phone use, electromagnetic fields, exposure to industrial and agricultural chemicals, infections, head trauma, immune status/allergies and genetic polymorphisms $(4,17,18)$. Nevertheless, little progress has been made so far to establish these environmental or endogenous factors as conclusive risk factors for gliomas.

The role of estrogenic exposure in reduced risk of gliomas has been suggested by several experimental and epidemiological studies (reviewed in (5)); however, the evidence has not yet become conclusive and more research is needed to draw definitive conclusions before hormone therapy can be developed for prevention or treatment of gliomas. This approach might be promising in the future, as several estrogen receptor beta $(\operatorname{ER} \beta)$ agonists have already displayed anti-glioma activity in vitro and in vivo (19). The present study aims to provide evidence to support the suppressive role of estrogenic exposure in the development of gliomas through epidemiological investigation of association between breast cancer and gliomas.
This study employs an established method for the analysis of the risk of multiple primary cancers (14) focusing on gliomas (and most prominently on glioblastoma) and invasive duct carcinoma of the breast. For the purpose of this study, the history of breast cancer diagnosis was considered as a marker of long-term estrogenic exposure. This approach is biologically plausible, since the exposure to endogenous or exogenous estrogens has been recognized as a risk factor involved both in early (20) and late stages of breast cancer development (21). For instance, women taking combined oral contraceptives (OC) display a small increase in the risk of breast cancer diagnosis that remains significant for 10 years after OC discontinuation (22). And similarly, women using hormone replacement therapy (HRT) displayed a higher risk of breast cancer diagnosis, which increased with duration of HRT and persisted for 4 years after cessation of the HRT use (23). Furthermore, postmenopausal women not using HRT displayed an increased risk of both $\mathrm{ER}+/ \mathrm{PR}+$ and ER-/PR- breast cancers with increased pre-diagnosis level of serum estradiol (24). Conversely, bilateral oophorectomy before the age of 40 years reduces the lifetime risk of breast cancer by approximately $75 \%$ (25). Likewise, the use of selective estrogen receptor modulator raloxifene reduces the risk of breast cancer diagnosis of ER+ breast cancer in postmenopausal women (26). Taken together, breast cancer diagnosis, especially in older women, can be considered as a surrogate marker for exogenous and/or endogenous long-term exposure to estrogens. This reasoning, together with the reported differences in the epidemiology, biology and clinical behavior of breast cancers diagnosed in younger and older women (11), motivated a design of this study, which determined the risk of brain cancers following breast cancer diagnosis separately for women aged 0-39 and 40+ years.

The finding of significantly reduced risk of brain cancers in women diagnosed with breast cancer at the age of $40+$ is consistent with the hypothesis that estrogenic exposure decreases the risk of development of gliomas. The strength of the evidence is supported by consistency of significantly decreased risk for several latency intervals after diagnosis of breast cancers and additionally by bi-directionality of this inverse association, because the risk of breast cancer was also found to be decreased in women diagnosed with glioblastoma.

In contrast, other investigators reported an increased risk of glioblastoma in women diagnosed with breast cancer in general ( $\mathrm{SIR}=1.16$; CI95: 1.04-1.29) and, more substantially, in women diagnosed before the age of 45 ( $\mathrm{SIR}=1.41$; CI95: 1.01-1.92) (27). The results of the present study are internally consistent, bi-directional and externally consistent with conclusions of other epidemiological as well as experimental studies on the possible inverse association between estrogenic exposure and gliomas. The association between breast cancers and gliomas has also been considered by other studies, mostly of case report (28) or case series (29) study types. These and other studies hypothesized the existence of association between the two cancers, caused possibly by hormonal, genetic and/or treatment effects, and implied a positive association, but limited number of cases and study design did not permit a quantitative corroboration or age stratification of the risk. This study is, to the best of its author's knowledge, the 
first study that determined an increased risk of brain cancers in female breast cancer patients diagnosed at $<40$ years together with a decreased risk of brain cancers in women diagnosed with breast cancer at the age $40+$ years. While decreased risk in older women is plausibly associated with estrogenic exposure, increased risk found in younger breast cancer survivors is most likely related to genetic factors, such as: germline pathogenic mutations of TP53 (Li-Fraumeni syndrome) or BRCA1 and BRCA2 genes. These genetic defects were associated with early onset breast cancers as well as gliomas (15). Indeed, young women diagnosed with breast cancer have greatly elevated risk of pathogenic germline BRCA mutations than older women $(30)$, and BRCA1 $(31,32)$ or BRCA2 (33) germline mutations were reported in glioma cases. Interestingly, two breast cancer cases with germline BRCA1 mutations, who later developed glioblastoma, maintained some level of BRCA1 expression in glioblastoma tissue; nevertheless, heterozygous inactivation of BRCA1 (31) could have played a critical role in glioblastoma development due to BRCA1 haploinsufficiency, which is known to cause genomic instability $(34,35)$.

The key assumption underlying this analysis is the independence of multiple cancers; therefore, misclassification of brain metastases of breast cancers as second primary brain cancers would undermine the validity of the results. This matter is of particular concern in context of epidemiology of brain cancers following breast cancers, because the half of the newly discovered brain tumors each year represent metastatic lesions, and breast cancers appear to be the second leading cause of brain metastases (36). However, misclassification of metastatic brain lesions as primary breast cancers would cause bias towards apparently increased risk of second brain cancers, while women aged $40+$ years displayed a decreased risk of subsequent brain cancers in this study, supporting validity of the results.

On the other hand, younger women diagnosed with breast cancers displayed an increased overall risk of brain cancers, which might be indicative of the risk of bias due to potential misclassification of the brain lesions. For this reason, diagnoses of brain cancers in young breast cancer survivors were reviewed, and 26 of 27 brain cancers were found to had been diagnosed by histopathology (13 glioblastomas, 3 anaplastic astrocytomas, 1 diffuse astrocytoma, 3 astrocytomas NOS, 4 oligodendrogliomas, 1 mixed glioma, 1 embryonal/primitive/medulloblastoma), which rules out bias due to misclassification. This result is indicative of a good quality of SEER 9 recorded data for second primary brain cancers in women diagnosed with breast cancer at younger age and suggests validity of the conclusion about an increased risk of second primary brain cancers in these women.

\section{Conclusion}

Breast cancer is the most frequently diagnosed cancer among women worldwide, with stable or increasing incidence and decreasing mortality in most high-income countries (37), which lead to a growing population of breast cancer survivors. Consequently, second primary cancers positively associated with breast cancer became an increasingly important public health issue. The results of this study suggested an increased risk of gliomas following breast cancers diagnosed in younger women, which warrants additional research on the early diagnosis and targeted prevention of gliomas in this group. Decreased risk of gliomas in women diagnosed with breast cancers at older age provides an additional evidence for the protective role of estrogenic exposure against gliomagenesis.

\section{References}

1. Travis LB. The epidemiology of second primary cancers. Cancer Epidemiol Biomarkers Prev 2006; 15 (11): 2020-2026.

2. Travis LB, Rabkin CS, Brown LM, Allan JM, Alter BP, Ambrosone CB, Begg CB, Caporaso N, Chanock S, DeMichele A et al. Cancer Survivorship - Genetic Susceptibility and Second Primary Cancers: Research Strategies and Recommendations. JNCI: J Nat Cancer Inst 2006; 98 (1): $15-25$.

3. Ostrom QT, Gittleman H, Liao P, Rouse C, Chen Y, Dowling J, Wolinsky Y, Kruchko C, Barnholtz-Sloan J. CBTRUS statistical report: primary brain and central nervous system tumors diagnosed in the United States in 2007-2011. Neuro Oncol 2014; 16 Suppl 4: iv1-63.

4. Bondy ML, Scheurer ME, Malmer B, Barnholtz-Sloan JS, Davis FG, Il'yasova D, Kruchko C, McCarthy BJ, Rajaraman P, Schwartzbaum JA et al. Brain tumor epidemiology: consensus from the Brain Tumor Epidemiology Consortium. Cancer 2008; 113 (7 Suppl): 1953-1968.

5. Cowppli-Bony A, Bouvier G, Rue M, Loiseau H, Vital A, Lebailly P, Fabbro-Peray P, Baldi I. Brain tumors and hormonal factors: review of the epidemiological literature. Cancer Causes Control 2011; 22 (5): 697-714.

6. Bethke L, Murray A, Webb E, Schoemaker M, Muir K, McKinney P, Hepworth S, Dimitropoulou P, Lophatananon A, Feychting M et al. Comprehensive analysis of DNA repair gene variants and risk of meningioma. J Natl Cancer Inst 2008; 100 (4): 270-276.

7. Pabalan N, Jarjanazi H, Ozcelik H. Association between BRIP1 (BACH1) polymorphisms and breast cancer risk: a meta-analysis. Breast Cancer Res Treat 2013; 137 (2): 553-558.

8. Wei RL, Ziogas A, Daroui P, Nangia C. Association Between Breast Cancer and Glioblastoma Multiforme in Women: A California Cancer Registry Population-Based Analysis. Internat J Radiat Oncol Biol Phys; 90 (1): S268.

9. Curtis RE, Freedman DM, Ron E, Ries LAG, Hacker DG, Edwards BK, Tucker MA, Fraumeni JF. New Malignancies Among Cancer Survivors: SEER Cancer Registries, 1973-2000, vol. 05-5302. Bethesda, MD: National Cancer Institute; 2006.

10. Rosen PP. Invasive mammary carcinoma. In: Diseases of the Breast. edn. Edited by Harris JR, E. LM, Morrow M, Hellman S. Philadelphia, PA: Lippincott-Raven; 1996: 393.

11. Gabriel CA, Domchek SM. Breast cancer in young women. Breast Cancer Res 2010; 12 (5): 212.

12. Schoenberg BS, Myers MH. Statistical methods for studying multiple primary malignant neoplasms. Cancer 1977; 40 (4 Suppl): 1892-1898.

13. Sahai H, Khurshid A. Confidence-Intervals for the Mean of a PoissonDistribution - a Review. Biometr J 1993; 35 (7): 857-867.

14. Curtis RE. New malignancies among cancer survivors : SEER cancer registries, 1973-2000. Washington, D.C.: U.S. Dept. of Health and Human Services, National Institutes of Health, National Cancer Institute; 2006. 
463-468

15. Kyritsis AP, Bondy ML, Rao JS, Sioka C. Inherited predisposition to glioma. Neuro Oncol 2010; 12 (1): 104-113.

16. Ostrom QT, Bauchet L, Davis FG, Deltour I, Fisher JL, Langer CE, Pekmezci M, Schwartzbaum JA, Turner MC, Walsh KM et al. The epidemiology of glioma in adults: a "state of the science" review. Neuro Oncol 2014; 16 (7): 896-913.

17. Gomes J, Al Zayadi A, Guzman A. Occupational and environmental risk factors of adult primary brain cancers: a systematic review. Int J Occup Environ Med 2011; 2 (2): 82-111.

18. Hanif F, Muzaffar K, Perveen K, Malhi SM, Simjee Sh U. Glioblastoma Multiforme: A Review of its Epidemiology and Pathogenesis through Clinical Presentation and Treatment. Asian Pac J Cancer Prev 2017; 18 (1): 3-9.

19. Lan Y-L, Zou S, Wang X, Lou J-C, Xing J-S, Yu M, Zhang B. Update on the therapeutic significance of estrogen receptor beta in malignant gliomas. Oncotarget 2017; 8 (46): 81686-81696.

20. Allred DC, Brown P, Medina D. The origins of estrogen receptor alpha-positive and estrogen receptor alpha-negative human breast cancer. Breast Cancer Res 2004; 6 (6): 240-245.

21. Dietel M, Lewis MA, Shapiro S. Hormone replacement therapy: pathobiological aspects of hormone-sensitive cancers in women relevant to epidemiological studies on HRT: a mini-review. Hum Reprod 2005; 20 (8): 2052-2060.

22. Collaborative Group on Hormonal Factors in Breast C. Breast cancer and hormonal contraceptives: collaborative reanalysis of individual data on 53297 women with breast cancer and 100239 women without breast cancer from 54 epidemiological studies. Lancet 1996; 347 (9017): 1713-1727.

23. Breast cancer and hormone replacement therapy. collaborative reanalysis of data from 51 epidemiological studies of 52,705 women with breast cancer and 108,411 women without breast cancer. Collaborative Group on Hormonal Factors in Breast Cancer. Lancet 1997; 350 (9084): 1047-1059.

24. James RE, Lukanova A, Dossus L, Becker S, Rinaldi S, Tjonneland A, Olsen A, Overvad K, Mesrine S, Engel P et al. Postmenopausal serum sex steroids and risk of hormone receptor-positive and -negative breast cancer: a nested case-control study. Cancer Prev Res (Phila) 2011; 4 (10): 1626-1635.

25. Feinleib M. Breast cancer and artificial menopause: a cohort study. J Natl Cancer Inst 1968; 41 (2): 315-329.
26. Cummings SR, Eckert S, Krueger KA, Grady D, Powles TJ, Cauley JA, Norton L, Nickelsen T, Bjarnason NH, Morrow M et al. The effect of raloxifene on risk of breast cancer in postmenopausal women: results from the MORE randomized trial. Multiple Outcomes of Raloxifene Evaluation. JAMA 1999; 281 (23): 2189-2197.

27. Wei RL, Ziogas A, Daroui P, Nangia C. Association Between Breast Cancer and Glioblastoma Multiforme in Women: A California Cancer Registry Population-Based Analysis, vol. 90; 2014.

28. Canda MS, Guray Durak M, Rs E, F A, Canda T. Association of Invasive Breast Carcinoma and Glioblastoma Multiforme: A Case Report with Histological and Immunohistochemical Features, vol. 34; 2004.

29. Piccirilli M, Salvati M, Bistazzoni S, Frati A, Brogna C, Giangaspero F, Frati R, Santoro A. Glioblastoma multiforme and breast cancer: report on 11 cases and clinico-pathological remarks. Tumori 2005; 91 (3): $256-260$.

30. Evans JP, Skrzynia C, Susswein L, Harlan M. Genetics and the young woman with breast cancer. Breast Dis 2005; 23: 17-29.

31. Boukerroucha M, Josse C, Segers K, El-Guendi S, Freres P, Jerusalem G, Bours V. BRCA1 germline mutation and glioblastoma development: report of cases. BMC Cancer 2015; 15: 181.

32. Elmariah SB, Huse J, Mason B, Leroux P, Lustig RA. Multicentric glioblastoma multiforme in a patient with BRCA-1 invasive breast cancer. Breast J 2006; 12 (5): 470-474.

33. Raufi A, Alsharedi M, Khelfa Y, Tirona M. Bilateral Triple-Negative Invasive Breast Cancer with a BRCA2 Mutation, and Glioblastoma: A Case Report and Literature Review. J Breast Cancer 2017; 20 (1): 108-111.

34. Konishi H, Mohseni M, Tamaki A, Garay JP, Croessmann S, Karnan S, Ota A, Wong HY, Konishi Y, Karakas B et al. Mutation of a single allele of the cancer susceptibility gene BRCA1 leads to genomic instability in human breast epithelial cells. Proc Natl Acad Sci U S A 2011; 108 (43): 17773-17778.

35. Salmena L, Narod S. BRCA1 haploinsufficiency: consequences for breast cancer. Womens Health (Lond) 2012; 8 (2): 127-129.

36. Harsh IV GR, Wilson CB. Neuroepithelial tumors of the adult brain. Philadelphia: WB Saunders; 1990.

37. DeSantis CE, Bray F, Ferlay J, Lortet-Tieulent J, Anderson BO, Jemal A. International Variation in Female Breast Cancer Incidence and Mortality Rates. Cancer Epidemiol Biomarkers Prev 2015.

Received April 4, 2018. Accepted April 9, 2018. 